\title{
Publisher Correction: Demise of faint satellites around isolated early-type galaxies
}

Changbom Park, Ho Seong Hwang (1D), Hyunbae Park and Jong Chul Lee

Correction to: Nature Astronomy https://doi.org/10.1038/s41550-017-0332-9, published online 1 January 2018.

Owing to a technical error, in the version of this Letter originally published the data points and their outlines in Fig. 1c were shifted relative to their correct positions. This has now been corrected. 